\title{
THYMIC CARCINOMA
}

Clin Biomed Res. 2016;36(3):176-177

1 Internal Medicine Division, Hospital de Clínicas de Porto Alegre. Porto Alegre, RS, Brazil.

2 Radiology Division, Hospital de Clínicas de Porto Alegre. Porto Alegre, RS, Brazil.

Corresponding author: Vitória Homem Machado vitoriahomemmachado@gmail.com Hospital de Clínicas de Porto Alegre Rua Ramiro Barcelos, 2350, sala 700 90035-903, Porto Alegre, RS, Brazil.
Vitória Homem Machadoํㅜ, Fernando Pivatto Júnior ${ }^{1}$, Felipe Soares Torres ${ }^{2}$

A 73-year-old man, previously asymptomatic and with no comorbidities, presented to the emergency department with a 3-month history of worsening dyspnea, lately to minimal efforts, associated with constitutional symptoms. Pulmonary auscultation showed reduced left lung sounds with expiratory wheezes. Laboratory evaluation was within normal limits. Chest x-ray demonstrated a large left-sided opacity associated with pleural effusion (figure 1A). Contrast-enhanced chest computed tomography (CT) confirmed the presence of a large, lobulated, and heterogeneous anterior mediastinal mass with central areas of calcification (figure 1B, C). The mass abutted the pericardium and chest wall and exerted mass effect on the heart and mediastinum. An ultrasound-guided transcutaneous biopsy was performed, identifying a carcinoma. Immunohistochemical evaluation diagnosed thymic carcinoma. Tumor resection was contraindicated due to risks related to injury of adjacent organs, procedure-related morbidity and high probability of incomplete tumor resection. Patient underwent four cycles of neoadjuvant chemotherapy with etoposide and cisplatin, without response. Radiotherapy was not performed due to anticipated high pulmonary toxicity in consequence of large dimensions of the radiation field. End-of-life care was then initiated. Patient remains alive and oligosymptomatic 4 months after the end of chemotherapy.

Thymic carcinomas are a heterogeneous group of aggressive, invasive epithelial malignancies. Their incidence is rare, occurring predominantly in middle-aged men. Most patients present with cough, shortness of breath, and chest pain. Fatigue, weight loss, and anorexia are common ${ }^{1}$. Thymic carcinoma is considered by some to be on a continuum with thymoma, with features of all tumor types seen in some patients. However, it is rarely associated with paraneoplastic disorders and demonstrates distant hematogenous metastasis more frequently. Because most patients with thymic carcinoma present with unresectable disease, this histology is associated with poor prognosis ${ }^{2}$. Radiographically, thymic carcinomas are heterogeneous with necrosis and calcifications and can be associated with pleural and pericardial effusions ${ }^{1}$.

The most common causes of anterior mediastinal masses include the following: thymoma, extragonadal germ cell tumors, thyroid disease, and lymphoma. Imaging plays an important role in establishing the location of the tumor as well as a possible invasion of adjacent structures, such as heart, pericardium, aorta and pulmonary artery. In most cases, age and imaging characteristics are helpful in narrowing the differential diagnosis ${ }^{3}$.

Thymomas are the most common neoplasm of the anterior mediastinum and appear on a chest radiograph as a well-defined lobulated mass in the anterosuperior mediastinum, typically anterior to the aortic root. Further evaluation with contrast-enhanced thoracic CT scanning usually reveals an encapsulated, well defined, soft-tissue mass, often with hemorrhage, necrosis, or cyst formation ${ }^{1}$. Germ cell tumors are a heterogeneous group of lesions containing tissues originating from primitive germ cell layers: ectoderm, mesoderm, and endoderm. Migration of primordial germ cells may allow these tumors to develop in an extragonadal location, such as the anterior mediastinum ( $15 \%$ of mediastinal masses in adults and $24 \%$ in children $)^{3}$. Teratomas are the most common germ cell tumor $(70 \%)$ and, on $\mathrm{CT}$, they frequently present 


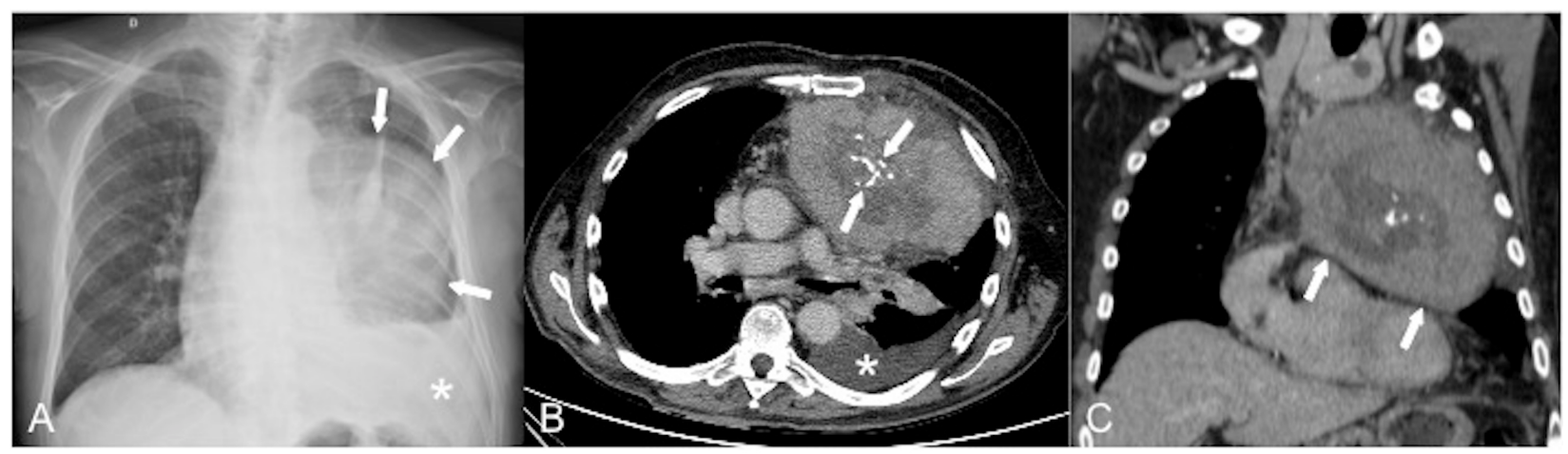

Figure 1: (A) Chest x-ray showing a large opacity (arrows) in the left hemithorax associated with left pleural effusion (asterix); (B) Transaxial contrast-enhanced computed tomography image showing a large anterior mediastinal mass with central calcifications (arrows) associated with left-sided pleural effusion (asterix); (C) Coronal reformatted computed tomography image demonstrating a large mass abutting the pericardium (arrowheads) and the chest wall.

as a lobulated cystic mass containing foci of fat (more than $75 \%$ ) and/or calcification (more than 50\%). An enlarged thyroid may descent to the anterior mediastinum and present as a mediastinal mass on chest radiograph. Intrathoracic goiter accounts for 3-6\% of mediastinal masses, with a majority located in the anterior compartment ${ }^{3}$. Cross-sectional imaging allows for defining the cervical extension of the mass and its connection to the thyroid gland, which is a key feature for differentiating a substernal goiter from other mediastinal masses. Lymphomas may also present as anterior mediastinal soft-tissue masses. About $50-70 \%$ of mediastinal lymphomas are due to Hodgkin disease, which has no sex predilection and shows a bimodal age distribution in young adults and patients older than 50 years ${ }^{3}$. On CT, lymphomas appear as homogeneous soft-tissue masses in the anterior mediastinum with surface lobulation and mildto-moderate contrast enhancement. Calcifications are uncommon in treatment-naive patients with lymphomas and should raise the possibility of an alternative diagnosis. Associated thoracic lymphadenopathy is common.

Here we showed the typical imaging findings of a thymic carcinoma. The combination of imaging characteristics with tumor location and patient age provides a roadmap for approaching the differential diagnosis.

\section{REFERENCES}

1. Duwe BV, Sterman DH, Musani Al. Tumors of the mediastinum. Chest. 2005;128(4):2893-909. PMid:16236967. http://dx.doi. org/10.1378/chest.128.4.2893.
2. Mikhail M, Mekhail Y, Mekhail T. Thymic neoplasms: a clinical update. Curr Oncol Rep. 2012;14(4):3508. PMid:22639107. http://dx.doi. org/10.1007/s11912-012-0246-8.
3. Shahrzad M, Le TS, Silva M, Bankier AA, Eisenberg RL. Anterior mediastinal masses. AJR Am J Roentgenol. 2014;203(2):W12838. PMid:25055288. http://dx.doi. org/10.2214/AJR.13.11998. 